\title{
Disparités et ambiguïté de l'accès aux ressources biologiques en Guyane française
}

\section{Guillaume Odonne et Damien Davy}

\section{(2) OpenEdition}

\section{Journals}

\section{Édition électronique}

URL : http://journals.openedition.org/elohi/794

DOI : 10.4000/elohi.794

ISSN : 2268-5243

\section{Éditeur}

Presses universitaires de Bordeaux

\section{Édition imprimée}

Date de publication : 1 janvier 2014

Pagination : 171-188

ISBN : 979-10-300-0021-4

ISSN : 2437-8175

Référence électronique

Guillaume Odonne et Damien Davy, « Disparités et ambiguïté de l'accès aux ressources biologiques en Guyane française », ELOHI [En ligne], 5-6 | 2014, mis en ligne le 01 janvier 2015, consulté le 01 mai 2019. URL : http://journals.openedition.org/elohi/794; DOI : 10.4000/elohi.794 


\title{
Disparités et ambiguïté de l'accès aux ressources biologiques en Guyane française
}

\author{
GUILLAUME ODONNE et DAMIEN DAVY \\ CNRS-Guyane USR3456
}

Dans cet article nous nous proposons de faire état des différentes positions, en Guyane française, des acteurs concernés par la problématique de l'accès aux ressources biologiques, sujet mis au goût du jour par la mise en place des APA (Accès et Partages des Avantages) dans ce département d'Outre-mer. Après avoir mis en exergue les opinions des différents acteurs (politiques, scientifiques, industriels et populations locales amérindiennes), nous détaillerons la complexité administrative concernant l'accès aux ressources. Dans un troisième temps, à travers des exemples concrets vécus par les Amérindiens de l'Oyapock (fleuve frontalier avec le Brésil situé à l'est du département), nous illustrerons la situation de ces peuples autochtones, qui sont ceux pratiquant le plus la nature et dépendant le plus de leur environnement pour leur vie quotidienne mais avec qui aucune concertation n'a été menée afin de permettre la pérennisation de ces pratiques.

Au-delà de l'utilisation de sources bibliographiques, cet article se veut le reflet de discours entendus par les auteurs à la fois lors de leurs enquêtes dans les villages de l'Oyapock mais, également, lors de différentes réunions impliquant tant des scientifiques que des politiques ou des acteurs de la vie économique locale.

Dans un premier temps, nous dresserons le contexte et passerons en revue le positionnement de ces différents acteurs.

\section{Ressources biologiques et mise en place des APA en Guyane}

La Guyane française est un DROM, c'est-à-dire un Département/Région d'Outre-mer (théoriquement une collectivité unique telle que définie par l'ar- 
ticle 73 dernier alinéa de la constitution), avec un statut politique très proche d'une région française, administrée par un conseil régional et un conseil général (qui devraient fusionner en 2015 pour laisser place à une assemblée unique). Elle est située sur le continent sud-américain. Si elle est dotée d'une façade maritime de plusieurs centaines de kilomètres sur l'Atlantique, nous ne nous intéresserons ici quà la partie terrestre. Celle-ci couvre $83846 \mathrm{~km}^{2}$, ce qui en fait la plus grande des régions ultrapériphériques de l'Union Européenne (et le plus grand département français). La frontière de l'est et du sud de la Guyane avec le Brésil constitue, avec ses 700 km, la plus longue frontière que la France possède avec un État étranger. Du point de vue écologique, la Guyane est un territoire megadivers, sinon un hotspot de biodiversité. De par la multiplicité des écosystèmes présents (mangroves, savanes, forêts primaires, inondables ou non, montagnes, inselbergs, etc.), la diversité floristique et faunistique est très élevée (Barret).

Le nombre d'espèces végétales est ainsi estimé à 5 500, dont plus d'un millier d'arbres, 1600 selon les dernières évaluations (Molino et al.). Environ 700 espèces d’oiseaux, 180 espèces de mammifères, 500 espèces de poissons, 130 espèces d'amphibiens et approximativement 160 reptiles y ont également été recensées (Charles-Dominique). On estime cependant que de nombreuses espèces restent à découvrir, notamment parmi les micro-organismes et les arthropodes.

La richesse de sa biodiversité n'est pas la seule caractéristique de la Guyane. La variété des cultures présentes en est une autre facette. On compte, classiquement, une quinzaine de groupes culturels importants : les créoles guyanais, descendants d’esclaves émancipés après l'abolition, forment aujourd'hui la première minorité de Guyane ; viennent ensuite les groupes marron, au nombre de cinq, issus de plusieurs vagues de marronnage dans les plantations du Suriname au XVIII ${ }^{e}$ siècle ; les personnes natives de la métropole ; les groupes amérindiens, au nombre de sept ; et les populations immigrées, originaires de Haïti, du Brésil, du Suriname, du Laos, du Pérou, etc. Parmi ces groupes culturels, plusieurs (essentiellement les Amérindiens et les Marrons) tirent, ou ont tiré un jour, leur subsistance de la forêt (Barret).

Cette biodiversité et la richesse des savoirs qui y sont liés font de l'accès aux ressources biologiques, tant aux niveaux scientifique quéconomique, et de leur utilisation au quotidien, un enjeu important en Guyane. C'est de cet accès que nous traiterons ici.

Suite à la convention sur la diversité biologique (Rio 1992), puis à la conférence de Nagoya (2010) et au protocole qui en découle (FRB 2011), la pertinence d'un mécanisme d'APA (appelé Access and Benefit Sharing en anglais) pour la Guyane s'est donc posée, avec un contexte un peu particulier. 


\section{Une situation paradoxale}

La Guyane possède, sensiblement, le même statut que les autres régions métropolitaines françaises. Les lois et règlements s'y appliquent de plein droit, mais deux aspects importants, sa biodiversité exceptionnelle, et la présence de " communautés autochtones et locales », la singularisent pourtant des autres régions et des autres DROM.

La France se considère comme un pays « utilisateur » autant que «fournisseur » de ressources génétiques ; il est donc envisagé un mécanisme d’accès aux ressources génétiques assez généraliste et piloté depuis la métropole (probablement avec un point focal au niveau du ministère de l'Écologie). Ce dispositif, prévu par le projet de loi-cadre sur la biodiversité (présenté à l'Assemblée nationale le 26 mars $2014^{1}$ ), devrait voir le jour dans les prochains mois. Or, le point de vue des autorités guyanaises (considérant la Guyane comme " fournisseur » avant tout) est tout autre.

La Guyane est très dépendante des importations, que ce soit dans le domaine des biens et services ou dans le domaine alimentaire. Elle connaît un faible développement économique, essentiellement basé sur les activités du centre spatial, la pêche à la crevette et les subsides de l'État. Le taux de chômage atteint les $22 \%$ de la population active (INSEE 2013). Le développement endogène souhaité par tous peine à voir le jour, malgré de forts investissements de l'Europe et de la France pour l'impulser (avec ce léger paradoxe d'un développement « endogène » impulsé par l'Europe ${ }^{2}$ ). L'idée que la riche biodiversité locale puisse devenir source de richesse et génère ce fameux développement a donc fait sa place chez les élus régionaux (SAR 2014 : 197). Ainsi, le congrès des élus a fait la demande auprès de l'État, en 2012, pour obtenir la compétence en matière d'accès aux ressources génétiques (dans le cadre de l'article 73 dernier alinéa de la Constitution, les collectivités peuvent demander des habilitations - sorte de transfert de légitimité - pour pouvoir légiférer dans des domaines particuliers).

\section{Le point de vue des personnalités politiques guyanaises}

Si l'on regarde et analyse le blason de la Guyane (Figure 1), on peut constater qu'il est assez représentatif de la vision qui prédomine aujourd'hui quant à la gestion de la biodiversité et à sa valorisation parmi les élites guyanaises. L'or

1. Voir http://www.assemblee-nationale.fr/14/projets/pl1847.asp, consulté le 25/09/2014.

2. Le paradoxe sur lequel nous attirons l'attention ici consiste en une volonté locale affichée de créer les conditions d'un développement économique endogène (SAR 2014 : 186) mais qui s'appuie grandement sur les aides venant de l'Etat (134 millions d'euros dans le cadre du Contrat de Plan État-Région 2007-2013) et de l'Europe (500 millions d'euros sur la période 20072013) sans compter la part importante du spatial dans le PIB de la Guyane (IEDOM 2013). 
occupe une place centrale, tant sur le blason que dans les politiques de développement (SAR 2014). La Guyane est un peu le Potosi des Français. Sur les cartes des côtes orientales de l'Amérique du sud au XVII ${ }^{e}$ siècle, le lac Parime, où l'on situait à l'époque l'Eldorado, se trouve ainsi en plein centre de la Guyane. La recherche de l'or a été le moteur de nombreuses explorations au cœur de ce territoire depuis au moins 300 ans. D’abord à travers le mythe de l'Eldorado, où les voyageurs ont cherché en vain de l'or en Guyane entre le $\mathrm{XVII}^{\mathrm{e}}$ siècle et le milieu du XIX ${ }^{\mathrm{e}}$ siècle, et ensuite, à partir des années 1850, avec la première ruée vers l'or qui suivit la découverte du précieux métal, et qui dura jusque vers les années 1950. Depuis le milieu des années

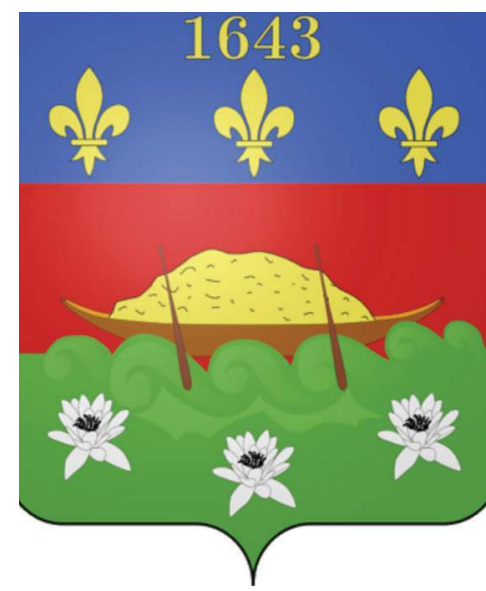

Figure 1 - Blason de la Guyane mettant en scène une pirogue chargée d'or. Source : Wikimedia commons. http://commons.wikimedia.org/ wiki/File:Blason_de_la_Guyane. svg? uselang=fr 1980 nous assistons à un deuxième cycle de l'or dans cette région (Oder). Ces éléments ont indéniablement marqué les esprits, l'exploitation aurifère pouvant être considérée comme un moteur historique du développement de la Guyane, au point d'influencer les contours du Parc Amazonien de Guyane (Fleury et Karpe). Dans son ouvrage de 1938, Retour de Guyane, Léon Gontran Damas proposait déjà de raser toute la forêt située sur la zone aurifère afin de développer la Guyane. La vision minière des ressources naturelles était déjà en place. Ce schéma pourrait être opposé, dans une certaine mesure et avec beaucoup de précautions, à celui de l'Amazonie brésilienne où les seringueiros (collecteurs de caoutchouc) et les castanheros (récolteurs de noix de Para) ont su faire accepter une vision de la valorisation de la forêt sous le prisme de l'extractivisme, impliquant une certaine durabilité. En Guyane, c'est la vision minière de la valorisation de la biodiversité qui prédomine : elle possède des ressources biologiques (des ressources génétiques selon la définition de la Convention pour la Diversité Biologique ${ }^{3}$ ), les industriels souhaitant les prélever versent une compensation financière. Il a d'ailleurs été écrit récemment, dans un document officiel du Conseil Régional préparant la loi-cadre sur la biodiversité, que la Guyane était un « réservoir de ressources génétiques »" illustrant cette

3. Voir le site de la convention pour la diversité biologique : http://www.cbd.int/convention/articles/default.shtml?a=cbd-02, consulté le 2 juin 2014.

4. Voir http://www.cr-guyane.fr/ressources/File/2013/juillet-aout-sept/contributions-regionales.pdf, consulté le 11 juin 2014. 
vision $^{5}$. Dans le Schéma d'Aménagement Régional de la Guyane pour les 20 prochaines années, en cours de validation, la valorisation économique de la biodiversité tient une place importante (SAR 2014).

Quant aux connaissances traditionnelles associées $\left(\mathrm{CTA}^{6}\right)$, le souhait qui émerge chez la plupart des politiciens locaux est celui d'une gestion, par le Conseil Régional, de l’ensemble des CTA pour les différentes communautés. La création théorique, en août dernier, d'un OBAG (Observatoire de la Biodiversité Amazonienne de Guyane) va dans ce sens. D’après les différentes présentations faites par la Région de cette structure lors de différentes réunions auxquelles nous avons participé, elle a pour objectif de fédérer les réseaux naturalistes, de venir en appui technique auprès des collectivités, d'impulser une dynamique de connaissance, de promouvoir la conservation des espèces, de favoriser la valorisation de la biodiversité, et enfin d'accompagner l'APA (le point focal au sens de la Convention pour la Diversité Biologique). Cet OBAG, sur des statuts d'association loi 1901, n’existe pour l'instant que sur le papier, et dès sa création théorique il est déjà mis à mal par les conflits politiques entre Conseil Régional et Conseil Général ${ }^{7}$. Il sera directement piloté par le Conseil Régional et représente, schématiquement, le relais entre collectivité d'une part, et scientifiques et industriels, d'autre part. Cette position s'inscrit, de plus, dans un contexte d'affirmation identitaire forte et d'autonomisation vis-à-vis de la métropole. En effet, si en 2010 les citoyens de Guyane française ont, lors d'un référendum statutaire, voté pour rester un département français, ils ont, par contre, voté pour une assemblée unique qui réunira les Conseils régionaux et généraux. Cette assemblée unique offrira une plus grande autonomie locale.

La volonté des collectivités locales concernant l'APA de gérer les CTA au nom de toutes les communautés de Guyane pose un problème à léchelon national, tant au niveau du ministère de l'Enseignement Supérieur et de la Recherche que du ministère de l'Ecologie, du Développement Durable et de l'Energie, où cette vision ne satisfait pas. Sur le fond, le projet de loi sur la biodiversité (projet de loi relative à la biodiversité $\mathrm{N}^{\circ} 1847$ du 26 mars 2014) va dans le sens d'une gestion centralisée des demandes et des décisions par un organisme non encore défini (et précisé par de futurs décrets), puis d’une éventuelle décentralisation concernant le partage des avantages, dans un second temps.

5. Voir également le journal France-Guyane du 25 novembre 2013 : http://www.franceguyane.fr/actualite/societe-social-emploi/le-developpement-par-les-plantes-179506. php, consulté le 25/09/2014.

6. Signifie qu'il existe un savoir traditionnel associé à telle ou telle ressource génétique. 7. En effet, le Conseil Général est dirigé par le partie indépendantiste MDES (Mouvement de Décolonisation et d'Émancipation Sociale) d’obédience de gauche, et la Région est dirigée par une équipe affiliée à l'UMP. 


\section{Le point de vue des scientifiques}

Le paradigme sur lequel repose la position la plus fréquemment rencontrée chez les scientifiques, et un certain nombre de naturalistes issus des associations de protection de la nature, est que la connaissance prime sur le développement économique, ou que tout du moins le deuxième n'est pas de leur ressort. Partant, l'idée de devoir demander une autorisation pour travailler n'est pas acceptée facilement par un certain nombre de scientifiques. Cela vient essentiellement de l'histoire de la recherche française. Les chercheurs (en sciences naturelles ou sciences humaines) ont, pour beaucoup, une vision de la connaissance pour la connaissance, sans objectifs d'applications ou de valorisation économique potentiels. De plus, comme les témoignages que nous avons recueillis le montrent, beaucoup craignent de voir ces demandes d'autorisation leur prendre un temps précieux, ce qui est d'autant plus gênant que le fonctionnement actuel de la recherche soriente vers une politique de projets avec des échéances souvent brèves. En conséquence, ils plébiscitent plutôt une option basée sur la simple déclaration du programme de recherche comme demande d'accès, et le ministère va également dans ce sens tant qu'aucune valorisation économique n'est prévue, comme l'explicite le projet de loi relatif à la biodiversité ${ }^{8}$. Cependant, les organismes de recherche nationaux implantés en Guyane (Centre National de la Recherche Scientifique, Institut de Recherche pour le Développement, Centre de coopération Internationale en Recherche Agronomique pour le Développement) participent aux réflexions liés aux APA avec les collectivités locales et le Parc Amazonien de Guyane.

\section{Le point de vue des industriels}

Les industriels sont assez prudents. Quelques industriels de la cosmétique ou de la pharmacie ont travaillé en Guyane dans les années 1980 et 1990, mais de manière ponctuelle et assez discrète. C'est toujours le cas aujourd'hui, dans la légalité, mais dans la discrétion. Étant donné le rôle de l'image pour ces industriels, il est facile de comprendre le risque qu'il y aurait à s'exposer inutilement. L'histoire de la salade coumarou a ainsi fait grand bruit autour de 2005 : une société de cosmétique souhaitait exploiter une plante (Mourera fluviatilis). Une première exploitation a eu lieu sur le fleuve Approuague où les riverains ont protesté, arguant d'un impact délétère sur l'ichtyofaune, d’autant plus contrariant que les poissons représentent une ressource alimentaire non négligeable.

8. « Tant que la R\&D ne débouche pas sur un produit ou un procédé commercialisable, l'acteur concerné pourra dans un premier temps procéder à une déclaration, puis dès que la perspective de commercialisation se précisera et en tout état de cause, avant la mise sur le marché, procéder à une demande d'autorisation ». Voir le site : http://www. assemblee-nationale.fr/14/projets/pl1847.asp, consulté le 20 octobre 2014. 
La société a donc ouvert une enquête publique pour le prélèvement de deux tonnes de cette plante sur le haut cours de la rivière Sinnamary, loin d'implantations humaines (Fleury et al., 2003). Lopinion publique locale, puis nationale, s'est émue de ce prélèvement problématique, eu égard à la productivité de cette espèce et à son rôle écologique encore mal compris, aussi, la société a décidé d’arrêter le prélèvement en Guyane9. La société en question nétait pas dans l'illégalité, mais le hiatus entre ce que la loi impose et ce que l'opinion publique perçoit aurait pu lui être néfaste en termes d'image. Depuis cet épisode, les industriels sont d'autant plus frileux que les politiques, ainsi que certaines associations de protection de la nature, se saisissent facilement du spectre de la biopiraterie (Aubertin et Moretti).

Au final, l'un des principaux freins à l'accès aux ressources génétiques par les industriels en Guyane est l'absence de protocole clair, garantissant une exploitation sereine. Plusieurs tentatives ont échoué devant l'impossibilité, pour certains interlocuteurs locaux, de traiter les demandes dans des délais raisonnables, de manière transparente et en accord avec la réalité industrielle (tonnage, contrôle qualité, fiabilité de l'approvisionnement, etc.).

\section{Le point de vue des communautés autochtones et locales}

Comme nous l'avons constaté lors des différentes réunions organisées sur ces sujets, les communautés autochtones et locales sont souvent oubliées dans les débats. De manière générale, qu'il s'agisse des groupes amérindiens ou des groupes marrons, le souhait le plus souvent rapporté est de pouvoir continuer à utiliser la forêt afin de poursuivre les activités socialement et culturellement marquantes que sont la chasse, l'agriculture sur brûlis, la pêche et la cueillette. Les prélèvements de ressources génétiques, dans ce cadre, sont assez peu nombreux, et il s'agit principalement de gibier, de poisson, de bois de construction, et du défrichement de zones limitées pour les terrains agricoles. Il existe, en Guyane, assez peu d'exploitation commerciale de la forêt, produits ligneux et produits non ligneux confondus. Mais il faut tout de même évoquer le discours, souvent entendu chez les populations de l'intérieur de la Guyane ${ }^{10}$, sur le pillage de «leurs » ressources, qui semble refléter davantage un discours politique local plutôt qu'une expérience véritable. Dans les faits, la plupart de nos interlocuteurs ne semblent pas y voir d'inconvénient si la communauté peut profiter de retombées économiques, même faibles, comme des emplois temporaires sur

9. Voir, sur le site blada, http://www.blada.com//boite-aux-lettres/infos-citoyennes/2466 Communique_de_Maiouri_Nature_Guyane.htm, consulté le 30 mai 2014.

10. Ces discours ont été recueillis auprès de nombreuses personnes lors de différentes missions de terrains effectuées dans les villages amérindiens de l'Oyapock et du haut-Maroni de 2009 à 2013. 
les expéditions, et tant que les ressources ne sont pas dégradées. L’exemple de prélèvements de cacao sauvage dans les forêts du haut Oyapock par le CIRAD (cas d'une recherche portant sur une ressource génétique sans Connaissances Traditionnelles Associées) est marquant : les Wayãpi se satisfaisaient dêtre rémunérés comme guides quand le Conseil Régional s'inquiète qu’on lui vole les gènes qui feront gagner des millions au CIRAD ${ }^{11}$. Les exemples d'utilisation à fins lucratives de RG avec CTA sont, à ce jour, inexistants en Guyane.

Le CCPAB (Conseil Consultatif des Populations Amérindiennes et $\mathrm{Bu}$ sinenge ${ }^{12}$ ), mis en place en Guyane française par le décret $n^{\circ} 2008-562$ du 17 juin 2008, a, quant à lui, rendu un avis le 8 décembre 2012 relatif au projet de charte du Parc Amazonien de Guyane ${ }^{13}$. Dans cet avis, le sujet des APA est abordé, et le $\mathrm{CCPAB}$ insiste sur la nécessité de lancer une réflexion concernant les rapports entre droit de propriété intellectuelle, droit individuel et droit collectif. Les droits collectifs constituent un sujet central pour les communautés autochtones de Guyane. Le CCPAB insiste également pour que les bénéficiaires des APA soient les populations autochtones ${ }^{14}$, et non des communautés d'habitants ou des communautés locales.

\section{L'accès aux ressources à des fins scientifiques ou commerciales, ou la complexité des strates administratives}

En France, de manière générale, l’accès physique aux ressources génétiques (nous nous limitons ici à ces dernières, excluant les CTA) est assez complexe. Avant même les protocoles de Rio ou Nagoya, il y avait, en effet, plusieurs

11. Nous avons été témoins de ces discussions ayant eu lieu dans le village wayãpi de $\mathrm{Zi}$ dock lors de la présentation de ce projet porté par le CIRAD en 2009. En fait, le CIRAD voulait faire des prélèvements décorce sur des pieds de cacaoyers sauvages poussant sur le haut-Oyapock, donc, en terre wayãpi. Ces prélèvements auraient servi à faire des recherches génétiques afin de trouver des cultivars résistants aux maladies qui déciment les plantations de cacaoyers en Afrique. Or, les Wayãpi n'utilisent pas du tout ces cacaoyers, et ne possèdent donc pas de savoirs associés à cette ressource, par contre ils sont les seuls à connaitre la localisation de ces végétaux sauvages. Le CIRAD a abandonné ce projet devant l'opposition politique locale.

12. Les Businenge, ou Noirs Marrons, sont les descendants d'esclaves rebellés durant la traite négrière et qui ont formés de nouveaux groupes ethniques comme les Aluku ou Boni, les Ndjuka et les Saramaka. Voir Price et Price 2003.

13. Voir http://www.97320.com/Avis-n-03-du-CCPAB-relatif-au-projet-de-charte-duParc-Amazonien-de-Guyane_a5747.html, consulté le 6 juin 2014.

14. Le CCPAB rappelle, dans son avis $n^{\circ} 3$ cité ci-dessus, que la France a ratifié l'article 8j de la Convention pour la Diversité Biologique reconnaissant ainsi l'existence de communautés autochtones et leurs droits liés aux savoirs et aux ressources. 
niveaux d'autorisations à obtenir. En Guyane particulièrement, la situation est compliquée par un enchevêtrement de réserves et de zones protégées ( $\mathrm{Li}$ narès et al.).

Sur les terrains privés, la question se règle de gré à gré entre le demandeur et le propriétaire. En Guyane, l'essentiel du foncier forestier est domaine de l'État, et donc géré par l'Office National des Forêts (ONF). Tout prélèvement végétal doit faire l'objet d'une demande et d'une autorisation de l'ONF comme la loi française le stipule (Davy et Filoche).

La Direction de l'Environnement, de l'Aménagement et du Logement centralise les demandes d'accès aux ressources dans une certaine mesure, quand il s'agit d'espèces protégées et/ou que les plantes doivent être prélevées dans des réserves. Les demandes faites à la DEAL ne dispensent cependant pas de faire les demandes à l'ONF.

Il faut y ajouter, le cas échéant, les réserves naturelles, au nombre de sept en Guyane, dont l'avis est incontournable, en fonction du lieu de collecte de la ressource.

En plus des conseils scientifiques de ces réserves, le CSRPN (le Conseil Scientifique Régional du Patrimoine Naturel) donne des autorisations concernant les espèces protégées. Ces autorisations sont importantes lorsque l'on collecte des espèces dont on ne connait pas a priori les déterminations scientifiques (c'est parfois le cas en ethnobotanique), et la DEAL encourage vivement les demandeurs à prendre contact, en amont, avec les conseils scientifiques des réserves et le CSRPN, afin d'obtenir des autorisations annuelles.

Pour les études réalisées sur le territoire du Parc Amazonien de Guyane, évidemment, le Conseil Scientifique du PAG doit être saisi. Avec deux réunions annuelles, les délais sont parfois conséquents. L’avantage étant que le PAG se propose, ensuite, de coordonner le transfert du dossier à la Région et au Département, puisque le décret de création du PAG, de 2007, le stipule ainsi ${ }^{15}$. Pour l'instant, si le PAG a déjà transmis des dossiers, les retours des Conseils Généraux ou Régionaux ne suivent que très lentement. D’un point de vue géographique, le parc est composé d'une zone de libre adhésion où le droit commun s'applique (n'importe qui peut venir y vivre, pêcher, chasser, etc.). Les droits de chasse, pêche, agriculture, habitation et cueillette sont garantis aux communautés. Le parc est également composé d'une zone de cœur, qui est une zone de forte conservation, où la pénétration est soumise à autorisation du directeur, sauf pour les communautés d'habitants du Parc. Les droits de chasse, de pêche, d'agriculture, d'habitation et de cueillette y sont reconnus aux seules

15. Pour consulter ce décret voir : http://www.legifrance.gouv.fr/affichTexte.do?cidTexte=JORFTEXT000000241891, consulté le 25/09/2014. 
communautés d'habitants. C'est le parc qui assume la cogestion de la Zone de Cour avec les communautés d'habitants (et non plus l'ONF).

La création de ZDUC (Zones de Droits d'Usage Collectifs), de concessions ou de cessions collectives ${ }^{16}$ est rendue possible depuis 1987 par le décret ministériel 87-267. Chaque zone est créée par un arrêté préfectoral spécifique. Il existe, à ce jour, dans toute la Guyane, 15 ZDUC, 9 concessions et 3 cessions collectives, représentant une superficie de 669686 ha, soit $8 \%$ de la superficie régionale (Davy et Filoche). Ces zones permettent un accès exclusif aux ressources pour les " communautés d'habitants qui tirent traditionnellement leurs moyens de subsistance de la forêt ", périphrase utilisée dans le décret 87267 évitant de nommer des ethnies, concept inconstitutionnel. Ce droit foncier permet, donc, de leur garantir l'accès à une terre (qui reste propriété de l'État) afin de perpétuer les pratiques d'agriculture sur brûlis, de chasse, de pêche et de cueillette. L'accès aux ressources ou à l'installation pour des personnes tierces doit faire l'objet d'une demande aux chefs coutumiers (qui sont des représentants nommés ou élus par les membres de la communauté).

Enfin, les autres autorisations, non liées aux ressources, mais nécessaires dans certains cas, concernent l'accès aux territoires du sud de la Guyane, réunis sous le terme de "Zone d'accès réglementé » (ZAR), et doivent être demandées à la préfecture qui consulte le Parc Amazonien de la Guyane et la mairie de la commune concernée. Intervient également la Commission Nationale Informatique et Libertés, car beaucoup deétudes en ethnobotanique associent des CTA à des noms d'informateurs et impliquent donc des dossiers supplémentaires de demandes d'autorisation.

\section{L'accès aux ressources par les populations autochtones de Guyane : de grandes disparités}

Le département de la Guyane est, aujourd'hui, le principal DROM habité par des populations autochtones. Il existe sept peuples amérindiens représentant quasiment 10000 personnes $^{17}$. À ceux-ci, il faut ajouter des communautés marronnes qui comptent plus de 40000 personnes (Price et Price). Nous ne parlerons, ici, que des peuples amérindiens. Ils vivent répartis sur le littoral

16. Ce dispositif législatif offre trois différents types d'accès au foncier : la ZDUC (pas de propriété mais un usufruit collectif octroyé à une communauté, illimité), la concession collective (pas de propriété, usufruit collectif octroyé à une personne morale comme une association, limité dans le temps) et enfin la cession collective (gratuite, propriété collective à une personne morale, possible à la fin de la période de concession collective).

17. Estimation de P. Grenand et D. Davy, non publiée. 
(pour les groupes kali'na, palikur et arawak-lokono) ou dans l'intérieur, sur le haut cours des fleuves Maroni (Wayana, Apalai et Teko) et Oyapock (Teko et Wayãpi) (voir Barret).

Les Amérindiens du littoral constituent la majorité de la population amérindienne avec près de 7000 personnes. Ils vivent principalement dans des villages, de plus en plus cernés par l'urbanisation, ces villages devenant petit à petit des quartiers périurbains. Du fait de leur plus grande proximité avec la société de consommation, leurs savoirs et savoir-faire sont largement mis à mal, mais un certain nombre de foyers continuent d'avoir une activité agricole, de chasse, de pêche, de cueillette ou une activité artisanale liée à des ressources naturelles (Davy et Filoche).

Dans l'intérieur, les quelque 1200 Wayãpi, 550 Teko et 1300 Wayana et Apalai vivent dans des villages forestiers sur des territoires qu'ils habitent depuis plusieurs siècles. Ils sont largement majoritaires sur leur territoire et, depuis 2007, les communes dans lesquelles ils vivent ont été intégrées au Parc Amazonien de Guyane. Une grande partie de l'alimentation de ces populations rurales est encore autoproduite via la chasse, la pêche ou l'agriculture (Davy et al. 2012).

En Guyane, depuis quelques années, l'accès aux ressources biologiques s'est compliqué pour les Amérindiens de l'Oyapock, et il existe un accès inégal à la terre ou aux produits forestiers entre les différentes communautés amérindiennes de l'Est (Davy et al. 2012). C'est ce paradoxe que nous allons illustrer maintenant.

\section{Trois Sauts}

La communauté vivant la plus en amont du fleuve Oyapock est celle des Wayãpi des hameaux de Trois Sauts (illustration I). Les quelque 600 personnes vivant dans ces villages descendent de Wayãpi arrivés à partir du début du XIXe siècle, puis par plusieurs vagues de migration jusque dans les années 1980 (Grenand $1982: 84$ et suivantes). Situés initialement plus en amont vers les sources du fleuve, les emplacements actuels des villages datent de la fin des années 1970. Tous ces villages sont, depuis 1994, inclus dans une ZDUC dont l'arrêté préfectoral de création précise que l'ONF délègue la gestion de la terre à la communauté (Davy et al., 2012).

La création du Parc Amazonien, en 2007, n’a pas remis en cause ces droits mais les a, au contraire, consolidés sur une plus grande étendue du territoire. A contrario, la création du Parc National des montagnes Tumucumaques, rive brésilienne, a de facto amputé ce territoire agricole de près de la moitié de sa superficie, puisque c'est toute la rive brésilienne et ses affluents qui ne sont plus accessibles à l'agriculture du fait du statut de conservation intégrale qui leur est conféré au Brésil. Cela signifie qu'aucune activité anthropique n’est 
théoriquement permise à l'exception de l'écotourisme. Seules les activités de chasse et de cueillette, plus fugaces, sont encore pratiquées par les Wayãpi de Trois Sauts au Brésil, mais de manière clandestine. Signalons qu'aucune discussion n'existe à ce sujet entre les deux États (Davy et al. 2012).

\section{Camopi}

Si l'on descend vers le Nord le cours de l'Oyapock pour se rendre dans le bourg de Camopi, on rencontre d'autres communautés wayãpi et teko (illustration I). Eux aussi, Amérindiens de nationalité française, vivent dans le périmètre du Parc Amazonien et sont bénéficiaires d'une ZDUC. Ils peuvent continuer leurs pratiques de subsistance sur la rive française, mais toute la partie brésilienne leur est également interdite pour l'agriculture, la chasse et la cueillette. Contrairement au haut Oyapock où la rive brésilienne n'est pas habitée, il existe face au bourg de Camopi un village de commerçants et de trafiquants brésilien, Vila Brasil. On en trouve un deuxième plus en aval, Ilha Bella, servant de base logistique au ravitaillement des garimpeiros $^{18}$. Depuis 2005, un poste de la police militaire brésilienne est implanté à Vila Brasil, et il exerce un contrôle sur la frontière. La présence permanente des commerçants, trafiquants et militaires empêche toute activité de prélèvement de plantes ou de gibier sur cette rive (Davy et al. 2012).

\section{St Georges}

Enfin, sur le bas-Oyapock, dans la commune de Saint-Georges de l'Oyapock, vivent des Amérindiens palikur (Illustration I). C'est un peuple transfrontalier vivant à cheval entre les deux rives de l'Oyapock, dont une moitié, environ, possède la nationalité française. Cette partie du bassin de l'Oyapock ne fait pas partie du Parc Amazonien de la Guyane. Les Palikur ne bénéficient d'aucune ZDUC, ce qui est dû à la fois à une opposition d'une ancienne équipe municipale et à des conflits internes au sein de la communauté palikur (Davy et Filoche). En l'absence de ZDUC, la gestion du foncier est donc toujours du ressort de l'ONF. Cette absence de ZDUC est une exception (partagée avec les Kali'na d'Iracoubo, les Palikur de Régina et les Kali'na de Mana) puisque la grande majorité des villages amérindiens de Guyane en bénéficie. Même si pour certaines communautés (les Arawak-Lokono de Sainte Rose de Lima

18. Le premier village composé de commerçants et de trafiquants est habité par 156 personnes tandis qu'Ilha Bela, base arrière de l'orpaillage illégal à destination de la Guyane, est peuplé de 400 personnes (Soares). Garimpeiro est un terme brésilien (et passé dans le langage courant en Guyane) désignant les orpailleurs clandestins, souvent originaires du Brésil. 
à Matoury ou les Palikur de Tonate-Macouria), leur ZDUC est difficilement accessible et très éloignée des villages - jusqu’à $50 \mathrm{~km}$ (ibid.).

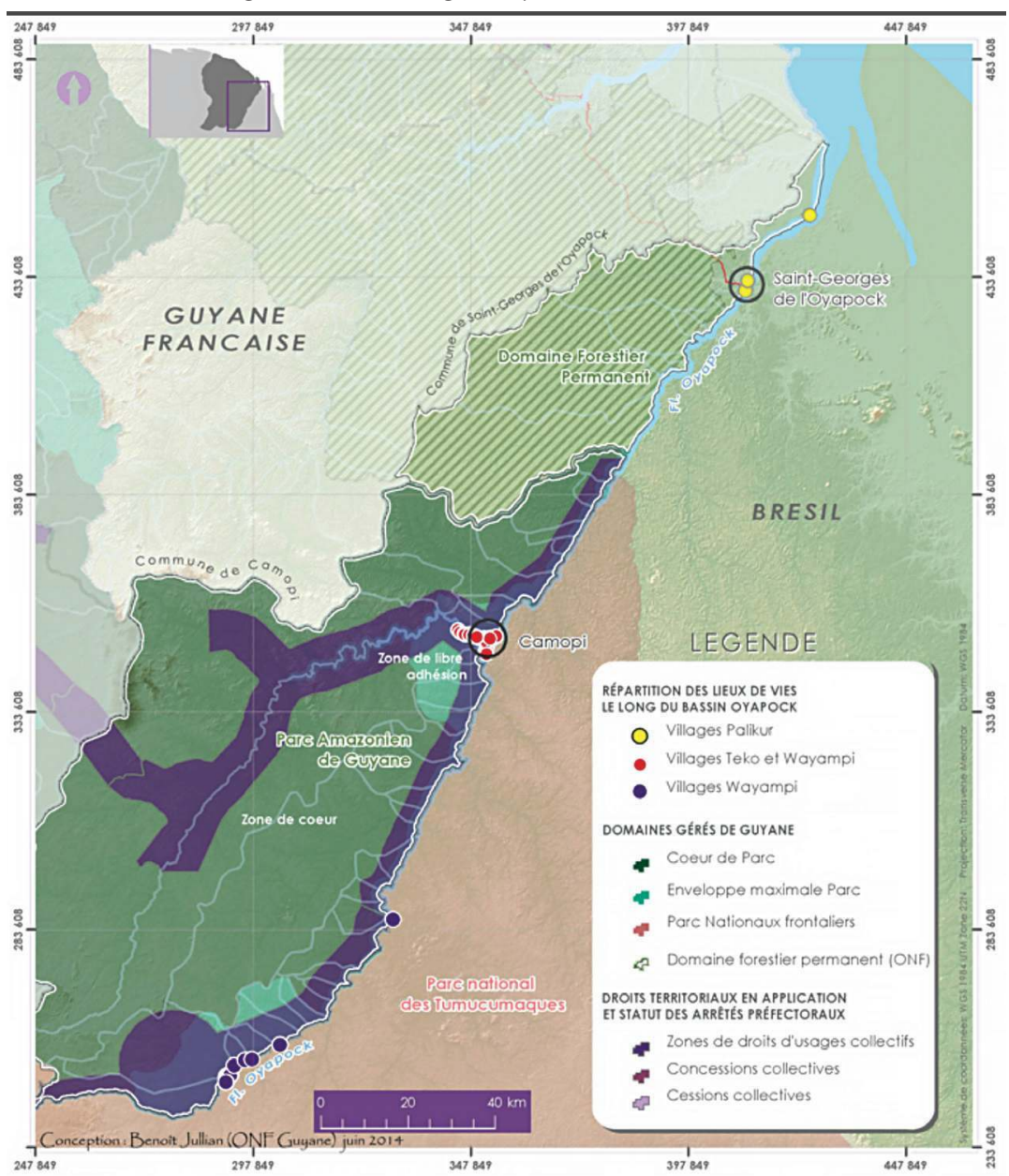

Illustration I - Localisation des zones de vies amérindiennes et des aires gérées dans la région de l'Oyapock, Guyane française (Conception Benoît Jullian, ONF, juin 2014)

Les Palikur possèdent, donc, des droits d'usages limités, et leurs zones agricoles, non définies officiellement, sont contraintes à la fois par l'urbanisation, une croissance démographique élevée et un Plan Local d'Urbanisme que la commune de Saint-Georges de l'Oyapock vient d’adopter en 2013, réduisant en conséquence leurs possibilités d'accès aux ressources. Le chef coutumier palikur a déposé une demande de ZDUC à la préfecture en 2012, afin de permettre aux membres de sa communauté de continuer la pratique de l'agriculture 
familiale. Les démarches administratives que cela implique sont des procédures auxquelles les Palikur doivent s'adapter. Précisons qu'aujourd'hui les Palikur constituent la majorité des agriculteurs pratiquant l'agriculture sur brûlis de cette commune (Koné).

La gestion par l'ONF a également des conséquences sur les modes de vies. L'exemple de l'utilisation des feuilles du palmier Toulouri (Manicaria saccifera) pour couvrir les habitats traditionnels des Palikur est symbolique. Les palmes sont prélevées sur un territoire géré par l'ONF qui fait payer les feuilles 30 centimes pièce. Un prélèvement gratuit pour des usages communautaires est tout de même toléré. Et cette situation prévaut pour toute coupe de bois (pirogues, maisons, etc.).Dans les faits, cela inhibe nombre de pratiques; certains vont voir l'ONF, d'autres fraudent (de bonne foi dans la mesure où ils se considèrent sur leur territoire) ; et d'autres vont moins fréquemment en forêt de peur des contrôles de police (Davy et Filoche).

Depuis la construction du pont transfrontalier, et en dépit du fait qu'il n'ait pas encore été inauguré et ne soit toujours pas en fonction, les effectifs de police ont fortement augmenté, ce qui a engendré de facto une augmentation des contrôles des habitants transfrontaliers (Davy et al. 2011). Certains Palikur se sont d'ailleurs vu confisquer des sacs de fruits de palmiers ou du gibier (sous prétexte de trafic). Les frustrations sont donc régulières et entrainent un fort sentiment d'abandon, et une impression que l'État français ne reconnaît par leurs droits ancestraux.

\section{Conclusion}

La France a la chance d'avoir sur son territoire des populations qui connaissent encore leur environnement d'une manière intime. Leurs connaissances du monde végétal ou animal sont d'une grande finesse et la Guyane a besoin de ces détenteurs de savoirs afin de combler de nombreuses lacunes dans la connaissance de cette formidable biodiversité. L'accès aux ressources est le pré-requis indispensable à la transmission des savoirs traditionnels liés à la biodiversité. Malheureusement, il nous semble que ces peuples sont trop souvent oubliés, ou consultés de manière trop succincte, et peu de choses sont faites pour consolider les droits des peuples autochtones quant à leur accès à la terre et aux ressources. Avec le décret de création du Parc Amazonien de Guyane de 2007, le décret ministériel de 1987 permettant aux populations amérindiennes de bénéficier de Zones de Droits d'Usage Collectifs, de concessions et de cessions collectives demeurent les seuls textes législatifs reconnaissant aux Amérindiens de Guyane française des pratiques et des modes de vie particuliers. Néanmoins, l'absence de statut national entraîne une grande complexité et surtout des iné- 
galités frappantes entre groupes culturels, allant de groupes dans le sud détenant la gestion de leur ZDUC (et donc l'accès privilégié à leurs ressources), et d'autres un peu dénués de tout, et dont l'accès aux ressources résulte plus de la tolérance que du droit.

Enfin, du point de vue institutionnel, des chercheurs ou des industriels, c'est encore un flou juridique. L'absence de réalité légale (l'OBAG n’existant que sur le papier, la loi-cadre sur la biodiversité arrivant au mieux au premier semestre 2014, ou le protocole de Nagoya nétant toujours pas en application) entretient de nombreuses incertitudes sur l'accès aux Ressources Génétiques. Cette situation fait fuir une partie des industriels et complique le travail des chercheurs dans la mesure où les lourdeurs politiques et administratives sont peu compatibles avec les financements sur projets. Au final, tout cela a plutôt tendance à dresser les acteurs les uns contre les autres.

\section{Financement :}

Les auteurs de ce travail ont bénéficié d'un financement "Investissement d'avenir " de l'Agence Nationale de la Recherche (CEBA, réf. ANR-10LABX-25-01).

\section{Bibliographie}

AUBERTIN Catherine, MORETTI Christian, «La biopiraterie entre illégalité et illégitimité ", in C. AUBERTIN, F. PINTON et V. BOISVERT, Les marchés de la biodiversité, Paris, IRD (Institut de Recherche sur le Développement), 2007, pp. 91-120.

BARRET Jacques (sous la dir. de), Atlas illustré de la Guyane, Paris, IRD, 2008, 219 p.

CHARLES-DOMINIQUE Pierre-Charles, La Guyane : Milieux, faune et flore, Paris, CNRS, 2011, 220 p.

DAMAS LÉON Gontran [1938], Retour de Guyane, Paris, Ed. Jean-Michel Place, coll. « Les Cahiers de Gradhiva », 2002, 126 p.

DAVY Damien, «Bois, fibres, feuilles, écorces, plumes... une culture matérielle végétale ", in G. MIGEON (sous la dir. de), Amérindiens de Guyane des cultures millénaires, entre les fleuves Approuague et Oyapock, Saint-Germain en Laye, Musée d'Archéologie nationale, 2010, pp. 58-73.

DAVY Damien, BOUDOUX D'HAUTEFEUILLE Madeleine, NICOLLE Sandra, GRENAND Françoise, "Du manioc et un pont : un Observatoire Hommes/Milieux sur la frontière francobrésilienne ", in J. L. Rebelo Porto, E. DoffSotta (sous la dir. de), Reformatações fronteiriças no platô das Guianas: 
(re)territorialidades de cooperações em construção, Rio de Janeiro, Publit, 2011, pp. 91-118.

DAVY Damien, TRITSCH Isabelle, GRENAND Pierre, " Construction et restructuration territoriale chez les Wayãpi et Teko de la commune de Camopi, Guyane française ", Confins, $\mathrm{n}^{\circ} 16,2012$, Disponible sur : http://confins.revues. org/7964, consulté le 25/09/2014.

DAVY Damien, FILOCHE Geoffroy (sous la dir. de), Zones de Droits d'Usage Collectifs, concessions, cessions en Guyane française : bilan et perspectives 25 ans après. Rapport détude coordonné par l'OHM (Observatoire Hommes-Milieux, Centre National de la Recherche Scientifique) Oyapock, Cayenne, 2014, 166 p.

FLEURY Marie, MORETTI Christian, BÉREAU Didier, "Usage des ressources forestières en Guyane : de la tradition à la valorisation », Revue Forestière Française, n ${ }^{\circ}$ 55, numéro spécial Guyane, 2003, pp. 291-305.

FLEURY Marie, KARPE Philippe, «Le parc national de Guyane : un arbitrage difficile entre intérêts divergents ", Journal de la société des américanistes, $\mathrm{n}^{\circ}$ 92-1 et 2, 2006, disponible sur: http://jsa.revues.org/3210, consulté le 25/06/2014.

FRB (Fondation pour la Recherche sur la Biodiversité), L'APA dans tous ses états. Paris, FRB, coll. " Des clés pour comprendre la biodiversité », Fiche clé $\mathrm{n}^{\circ} 2$, mars 2014, disponible sur http://www.fondationbiodiversite.fr/images/ stories/telechargement/fiche_cles_apa_web.pdf, consulté le 10/11/2014.

GRENAND Pierre, Ainsi parlaient nos ancêtres : essai dethnohistoire wayãpi, Paris, ORSTOM (Office de la Recherche Scientifique et Technique Outre-Mer), 1982, $420 \mathrm{p}$.

IEDOM (Institut d'Émission des Départements d'Outre-Mer), Guyane. Rapport annuel 2013, Paris, Iedom, 2013, 203 p., disponible sur http://www.iedom. fr/IMG/pdf/ra2013_guyane_avec_liens_sommaire.pdf, consulté le 25/09/2014.

INSEE Guyane-Antilles (Institut National de la Statistique et des Études Économiques), "Bilan économique 2012 en Guyane", Antiane éco. La revue économique des Antilles-Guyane, $\mathrm{n}^{\circ} 76,2013$, disponible sur le site : http://www.insee. $\mathrm{fr} / \mathrm{fr} /$ insee_regions/guyane/themes/ae_bilan/aes76gy/aes76gy.pdf consulté le 25/09/2014.

KONÉ Tchansia, « L'agriculture à Saint-Georges de l'Oyapock : bilan et perspectives ", Confins, $\mathrm{n}^{\circ}$ 16, 2012, disponible sur : http://confins.revues.org/8045, consulté le 25/09/2014.

LINARÈS Sébastien (sous la dir. de), Atlas des sites et espaces naturels protégés de Guyane, Cayenne, DIREN (Direction Régionale de l'Environnement) Guyane, 2007, 96 p.

MOLINO Jean-François, SABATIER Daniel, PRÉVOST Marie-Françoise, FRAME Dawn, GONZALEZ Sophie, BILOT-GUÉRIN Véronique, Liste des espèces d'arbres de la Guyane Française. Rapport final de la Convention E 24/08, 
Cayenne-Montpellier, Ministère de l'Agriculture et de la Pêche - IRD, 2009, $59 \mathrm{p}$.

ODER Jessica, «Vers la structuration d'une filière aurifère durable ? Étude du cas de la Guyane française ", EchoGéo, no 17, 2011, disponible sur : http:// echogeo.revues.org/12587, consulté le 25/09/2014.

PRICE, Richard et PRICE Sally, Les Marrons, Chateauneuf-le Rouge, Vents d'ailleurs, 2003.

SAR. Schéma d'Aménagement Régional de la Guyane : projet de SAR arrêté le 15 janvier 2014. 2014, 454 p., disponible sur : http://www.cr-guyane.fr/ ressources/File/2014/jan-fev-mar/projet-SAR-arrete_15012014.pdf, consulté le $25 / 09 / 2014$.

SOARES, Christiani, Vila Brasil, Ilha Bela e Camopi: efeitos da migração para os garimpos da fronteira franco-brasileira, Master 2 en Développement régional, Université Fédérale d’Amapa (Brésil)/OHM, 2013, 169 p.

Résumé : Le territoire de la Guyane possède la particularité d'être la seule région française (sans compter les Territoires d'Outre-mer) où vivent des populations autochtones possédant encore de nombreux savoirs locaux liés à la nature avec des savoir-faire associés. Faisant de la France à la fois un utilisateur potentiel de ressources génétiques mais aussi un fournisseur de ces mêmes ressources et de connaissances traditionnelles associées (CTA), la Guyane française tient une place particulière. Nous nous proposons ici de discuter de la mise en place de l'accès et partage des avantages (APA) en Guyane, mais surtout de montrer le positionnement des différents acteurs (politiques, scientifiques, industriels et populations locales amérindiennes) sur ce sujet. Nous insisterons plus particulièrement sur l'ambigüité de l'accès aux ressources biologiques chez les peuples amérindiens de la région de l'Oyapock, dans l'est de la Guyane française.

Mots clefs : Guyane française, accès et partage des avantages (APA), Oyapock, ressources biologiques, Amérindiens.

\begin{abstract}
French Guiana is a French overseas territory that has the particularity to be the only French region (stricto sensu) where indigenous people are still living. These people are holders of traditional ecological knowledge and due to their presence and the rich biodiversity of the Amazonian forest, France is not only a potential user of genetic resources, but also a provider of the latter, along with associated traditional knowledge (ATK). It gives a special importance to French Guiana. We propose here to explain how the Access and Benefit Sharing (ABS) processes will be put in place in French Guiana and how the local actors (politicians, scientists, industrialists or Amerindians) are positioning themselves. We will insist on the ambiguity and the difficulties for the Amerindians of the Oyapock region (eastern French Guiana) to access the biological resources.
\end{abstract}

Keywords : French Guiana, access and benefit sharing (ABS), Oyapock, biological resources, Amerindians.

Guillaume Odonne (ethnopharmacologue) est docteur en Pharmacie, titulaire d'un DEA de biologie tropicale, et enfin d'une thèse de Doctorat en Chimie des substances naturelles, aujourd'hui postdoc au CNRS Guyane. Guillaume Odonne a toujours travaillé dans le champ de l'ethnopharmacologie amazonienne. Entre le Suriname, le Pérou ou la Guyane française, ses travaux de terrain avec des populations marronnes ou amérindiennes l'ont amené à se pencher particulièrement sur le rapport que les populations autochtones entretiennent avec leurs plantes médicinales. Entre perceptions émiques, pratiques empiriques, activités biologiques et explications phytochimiques, c'est l'étude 
de cette relation dynamique Homme-plantes-maladies qui le passionne. En parallèle de cela, la problématique de l'accès aux ressources, que cela soit pour les Amérindiens avec lesquels il travaille ou dans le cadre de ses travaux en tant que chercheur, ont pris une importance considérable ces dernières années. Cet article a été écrit en forme de témoignage des réalités croisées qui se côtoient en Guyane.

With a doctorate in Pharmacy, a master's degree in Tropical Biology and a Ph. D. in Phytochemistry, now researcher at the French CNRS, Guillaume Odonne always worked in the field of Amazonian ethnopharmacology. In Surinam, Peru and French Guiana, his fieldwork with native populations led him to take an interest in the relationship native people have with their medicinal plants. From emic perceptions and empirical practices to pharmacological activities and phytochemical rationalisation, he studies the dynamic links between people, plants and diseases. On the other hand, the issues of access to biological resources, either for the Amerindians he works with, or for his own lab-work, took a considerable importance in the last years. This paper was written as a testimony of the different realities observed in French Guiana.

Damien Davy est ethnologue et ingénieur de recherche au CNRS-Guyane (USR 3456), il est actuellement directeur de l'Observatoire Hommes-Milieux Oyapock. Vivant en Guyane française, il mène depuis plus de dix ans des recherches sur l'ethnobotanique, les savoirs locaux et les relations que les populations amérindiennes entretiennent avec leur environnement, leur territoire. Récemment il a mené des recherches sur les changements socio-démographiques et les problématiques foncières des populations vivant sur le bord du fleuve Oyapock.

Damien Davy is an anthropologist and a research engineer at CNRS-Guyane (USR 3456), and he is currently the director of the observatory Human-Environment Oyapock. Living in French Guiana, he has led research on ethnobotany, local knowledge and relationships between Amerindian peoples and their environment and their territory for more than ten years. Recently he has conducted research on the socio-demographic changes and land issues of people living on the edge of Oyapock River. 